\title{
On the Choice of the Geometrical Extrapolation Models for the Mg-Al-Sr System Based on Experimental Investigation
}

\author{
M. Aljarrah ${ }^{a}$, M. Omari ${ }^{b *}$ (1), A. Alkhazali ${ }^{a}$ \\ ${ }^{a}$ The Hashemite University, Faculty of Engineering, Industrial Engineering Department, Zarqa, Jordan \\ ${ }^{b} J o r d a n$ University of Science and Technology, Mechanical Engineering, Irbid, Jordan
}

Received: June 27, 2020; Revised: August 23, 2020; Accepted: October 7, 2020

\begin{abstract}
In the current work, solidification curves were deduced experimentally from Differential Scanning Calorimetric (DSC) using heat transfer model, then compare our experimental work with different extrapolation solidification curves models predicted based on thermodynamic properties of binary sub-systems. The motivation of this work is to better predict ternary thermodynamic properties for binary phase diagram and the error of predicting arises from misjudging the asymmetric component in the asymmetric geometric model, It was found that Kohler gives a better agreement than Muggianu (symmetric model). Tested alloys show that, choosing strontium $(\mathrm{Sr})$ element as the asymmetric component in Mg-Al-Sr system gives the best prediction for solidification curves. In addition, the solidification curves calculated using Toop model for the ternary system ( $\mathrm{Al}$ or $\mathrm{Mg}$ is asymmetric) shows the poorest fit solidification curves construed from DSC data.
\end{abstract}

Keywords: Symmetric/asymmetric model, Heat transfer model (HTM), DSC.

\section{Introduction}

Equilibrium phase(s) at certain temperature and composition can be described by phase diagram. Material scientist has been using phase diagram as an essential tool for alloy development and material processing. However, constructing experimental phase diagram is costly and time-consuming. In 1973, different thermodynamic modeling of phase diagram was discussed during the first CALPHAD meeting ${ }^{1}$, this established the basic methods for calculating binary phase diagrams with a high accuracy based on key experiment. Besides, thermodynamic properties of a ternary solution can be constructed based on well-established binary sub-systems.

Thermodynamic properties of a ternary system can be evaluated from critically evaluated thermodynamic data of the binary sub-components using various geometrical methods ${ }^{2,3}$. Pelton ${ }^{4}$ thoroughly proposed different geometric models to evaluate thermodynamic properties of a ternary solution. To obtain an accurate ternary system, a precise extrapolation models should be carefully chosen. Geometric extrapolation models are divided into two treatments: symmetric $^{5,6}$ and asymmetric ${ }^{7,8}$ models are the most common. In order to correctly predict ternary system, it is important to choose the appropriate symmetric/asymmetric model. Many researchers ${ }^{4,-14}$ paid a great attention to choose the suitable geometrical model, symmetric or asymmetric, that presents the ternary solution. Pelton ${ }^{4}$ and Chartrand and Pelton ${ }^{13}$ analytically found that if two components are chemically similar but different from the third component then, an asymmetric model is physically more appropriate than symmetric model. Whereas, Qiao et al. ${ }^{14}$ studied the similarity of molten salt and alloy ternary systems based on the enthalpy of mixing of the binary subsystems, they suggested that if A-B and B-C are thermodynamically similar and different from $\mathrm{A}-\mathrm{C}$ then $\mathrm{B}$ is to be considered

*e-mail: engomari@just.edu.jo as the asymmetric component in the A-B-C ternary system. To the best of our knowledge, there are no published studies on the choice of extrapolation model using experimental techniques. The main novelty of the proposed approach is the attempt to choose the proper geometrical extrapolation model based on the experimental investigation.

\section{Method of Determining the Solidification Curve}

Solidification curves were obtained from Differential Scanning Calorimetric (DSC) measurements and compared with the calculated solidification curves using different extrapolation techniques; based on Heat transfer model. The experimentally deduced solidification curves from DSC measurements will be compared with the calculated solidification curves using different extrapolation models. This shall resolve many speculations pertinent to reasonably choose the correct symmetric/asymmetric extrapolation model.

Solidification curves will be established from the DSC measurements using Tian equation taking into account the heat transfer between the specimen and the reference ${ }^{15-18}$.

The heat flow rate produced inside the sample (reaction, transition) is time dependent and can be expressed as follow:

$q_{r}=-q-\left(C_{s}-C_{r}\right) \frac{d T_{r}}{d t}-r_{f s} C_{s} \frac{d q}{d t}$

Where,

$\mathrm{q}_{\mathrm{r}}$ : Heat flow produced inside the sample (reaction, transition) $\mathrm{q}$ : is the heat flow difference between the sample and the reference which is directly measured by the DSC; $t$ is the time; $T_{r}$ is the temperature of the reference

$C_{s}$ and $\mathrm{Cr}$ are the heat capacity of the sample and reference, respectively.

Further details of heat transfer model could be found in ${ }^{15,18-20}$. 


\section{Materials and Methods}

The investigated magnesium alloys were cast using high purity elements $(99.8 \mathrm{wt} \% \mathrm{Mg}, 99.999 \mathrm{wt} \% \mathrm{Al}$ and $99.5 \mathrm{wt} \%$ $\mathrm{Sr}$ ) in an induction furnace under argon with $1 \% \mathrm{SF} 6$; since Magnesium is highly flammable and reactive; $\mathrm{Mg}$ reacts with water at room temperature. Therefore, flowing argon is used with $1 \%$ SF 6 to protect the melt from oxidation, samples are listed in Table 1. The actual chemical composition of the studied samples was quantitatively measured by Inductively Coupled Plasma (ICP) atomic emission spectrometry. Mass loss was below $4 \%$ for all studied samples. Choosing those alloys is because of Magnesium is the lightest of all metals used as the basis for structural alloys. This makes magnesium alloys attractive for transportation applications such as automobiles and airplanes for weight reduction and higher fuel efficiency. Mg-6Al-2 Sr (AJ62) is used for application in transmission cases and oil pans.

Table 1. Examined samples with their compositions

\begin{tabular}{cccc}
\hline Sample & Sr (wt.\%) & Al (wt.\%) & Mg (wt.\%) \\
\hline 1 & 3.32 & 9.39 & 87.29 \\
\hline 2 & 7.09 & 18.09 & 74.82 \\
\hline 3 & 6.88 & 27.67 & 65.45 \\
\hline 4 & 4.56 & 31.63 & 63.81 \\
\hline
\end{tabular}

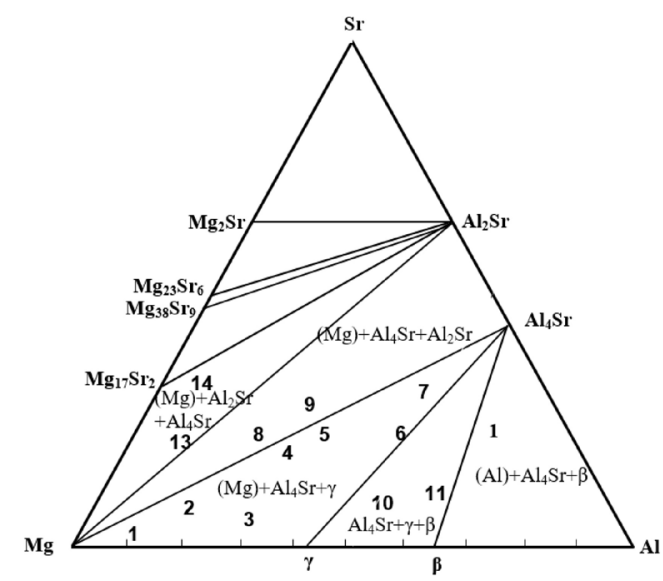

Figure 1. Isothermal section of Mg-Al-Sr ternary phase diagram at $25^{\circ} \mathrm{C}$ with the studied samples ${ }^{21}$.

(a)

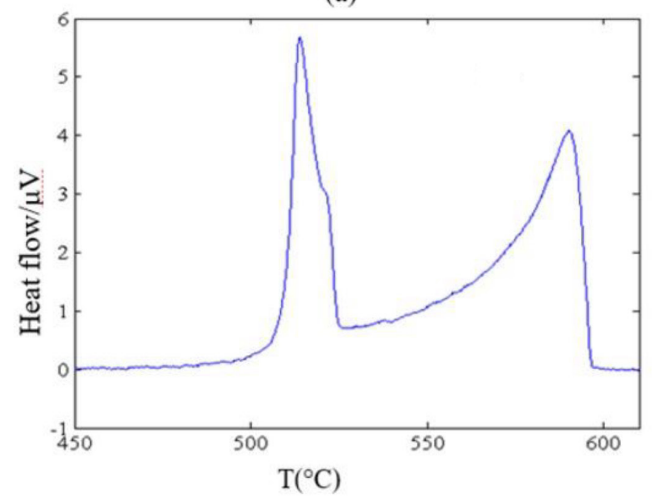

Thermal history of the investigated alloys was achieved using a Setaram Setsys DSC-1200 instrument to obtain solidification curves using Heat-transfer model using Tian equation ${ }^{15-18}$. The DSC equipment was calibrated for temperature using pure Magnesium and Aluminum elements. The studied samples (weight between $42-58 \mathrm{mg}$ ) were cut and manually polished to remove any possible contaminated surface layers. Acetone has been used to clean alloys, and then place them in a graphite crucible with a lid cover to contain Magnesium vapors and protect the DSC apparatus. DSC chamber was purged with pure argon twice to avoid oxidation. The DSC apparatus was run with rates of $4^{\circ} \mathrm{C} / \mathrm{min}$.

Solidification curves were calculated using FactSage software from thermodynamic database of binary sub-systems of Mg-Al-Sr ternary phase diagram ${ }^{19-21}$.

\section{Results and Discussions}

The calculated Isothermal section of Mg-Al-Sr ternary phase diagram at $25^{\circ} \mathrm{C}$ with the studied samples are shown in Figure 1. Sample 1 is located in $(\mathrm{Mg})+\mathrm{Al}_{4} \mathrm{Sr}+\gamma$ phase region. DSC spectra of sample 1 (3.32/87.29/9.36, Sr/Mg/Al, wt.\%) through cooling is shown in Figure 2. Comparison between solidification curves calculated from different extrapolations models and that deduced from DSC are shown in Figure 3. Solidifications curves calculated from different extrapolation techniques and deduced from DSC show similar trends.

Around $50 \%$ of solid fraction is approximately predicted from different extrapolation models except Muggianu model as observed in Figure 3. Note that at the end of the solidification, the amounts of the secondary phases determined by DSC and modeling fall close to Toop model when $\mathrm{Sr}$ was singled out, and Kohler model (symmetric) as can be seen in Figure 3. This finding is in accord with ${ }^{13}$ works where they found that in a dilute solution, Kohler model is the preferred model.

Figure 4 shows that $15 \%$ of solid fraction calculated from Muggianu and Toop ( $\mathrm{Mg}$ is singled out) fall approximately to that fraction deduced from DSC. Whereas, Kohler and Toop ( $\mathrm{Sr}$ is singled out) solidification curves are close to experimentally solidification curves from $15 \%$ to $45 \%$ solid fraction. From $45 \%$ solid fraction to solid state all geometric model is approximately predict solidification pattern except Toop (Al is singled out).

Sample 3 is located in $(\mathrm{Mg})+\mathrm{Al}_{4} \mathrm{Sr}+\gamma$ phase region, SEM image and solidification curves are shown in Figure 5.

(b)

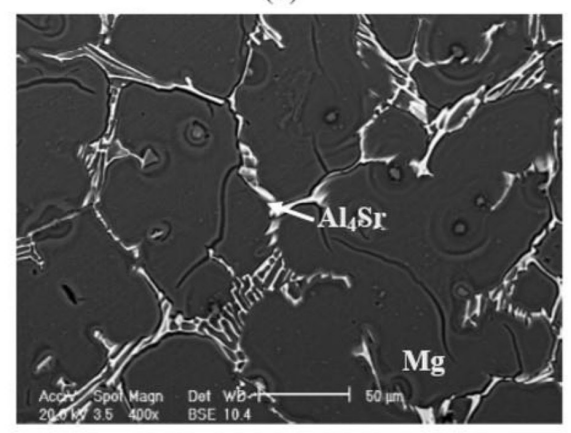

Figure 2. (a) DSC spectra and (b) SEM for sample 1 (3.32/87.29/9.39, Sr/Mg/Al, wt.\%). 
As shown in Figure 5, the calculated curves of solidification from geometric model, Kohler and Toop ( $\mathrm{Sr}$ is singled out), predict approximately solidification behavior of alloy 3 . The figure shows that, after $60 \%$ of solid fraction, all geometric model falls close to each other. In sample 4, the geometric model of Kohler and Toop ( $\mathrm{Sr}$ is singled out) gives the best estimation of solidification curve of alloy 4 especially when

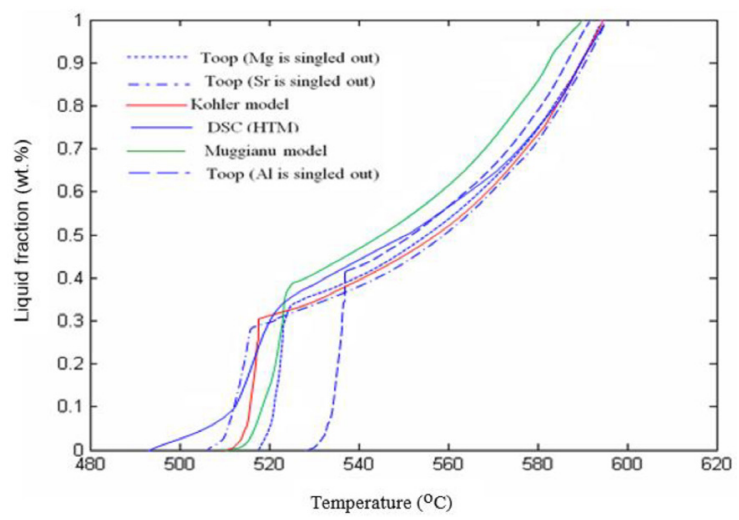

Figure 3. Solidification curves of sample 1(3.32/87.29/9.39, $\mathrm{Sr} /$ $\mathrm{Mg} / \mathrm{Al}$, wt.\%).

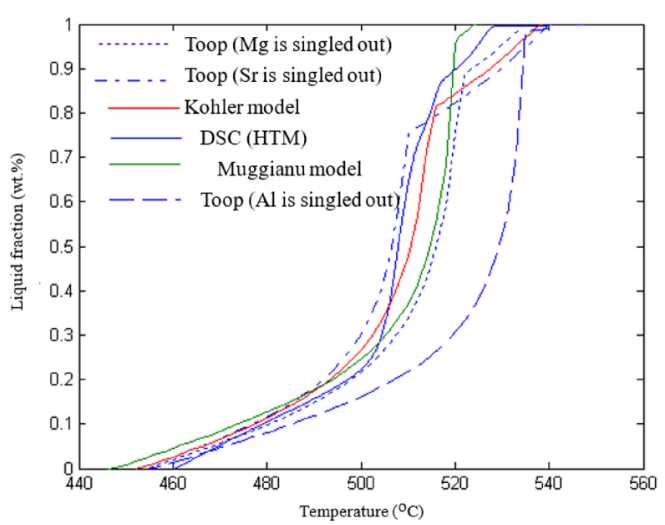

Figure 4. Solidification curves of sample 2 (7.09/18.09/74.82, $\mathrm{Sr} /$ $\mathrm{Al} / \mathrm{Mg}$, wt.\%). solid starts to form. However, the other geometric model shown in Figure 6 falls close to the experimental curve.

In comparison between Muggianu and Kohler, Kohler model gives better predication for solidification Curves compared to that calculated by Muggianu model. since Kohler assumes that at constant $\mathrm{X} 1 / \mathrm{X} 2$ ration the energy change of $\alpha_{12}$ of the pair exchange is constant, while Muggianu model assumes the energy equals to its value at the geometrically closet to the binary composition. Although $\mathrm{Mg}-\mathrm{Al}$ and $\mathrm{Mg}-\mathrm{Sr}$ systems are thermodynamically similar, treating $\mathrm{Sr}$ in a single group (Toop asymmetric) approximately predict solidification curve than that of treating $\mathrm{Mg}$ or $\mathrm{Al}$, which is in accord with ${ }^{13,22}$. Based on the above findings, solidification curves calculated from Kohler and Toop (treating $\mathrm{Sr}$ as singled out) geometric model give the most suitable solidification curve. Since, $\mathrm{Mg}$ and $\mathrm{Al}$ are chemically similar to each other but different from $\mathrm{Sr}$, then using $\mathrm{Sr}$ as singled out is the best geometric asymmetric model. Moreover, Toop model with an asymmetric component $\mathrm{Al}$ or $\mathrm{Mg}$, this was arbitrary chosen, provided the poorest fit with DSC data as can be seen in Figures 2-6. Therefore, using the geometric model to evaluate the thermodynamic properties of ternary solution from optimized data for the binary subsystems, the idea is to reasonably choose asymmetric model or/and asymmetric model, in which the thermodynamic asymmetric component should be precisely determined.

\section{Conclusion}

Several thermodynamic models have been proposed by different researchers for calculation of the ternary systems from its optimized sub-binary system. Each one of the models has its own method to represent the ternary system. Hence choice of the ternary extrapolation model plays a vital role to represent the ternary system. Two symmetric (Kohler and Muggianu) models and one asymmetric (Toop with three different combinations) model have been applied to calculate solidification curves of the Mg-Al-Sr system. These curves were compared to solidification curves deduced from DSC curves. Kohler and Toop (where $\mathrm{Sr}$ is single out) give the best fit to the solidification curves deduced experimentally from DSC curves in current composition ranges. (a)

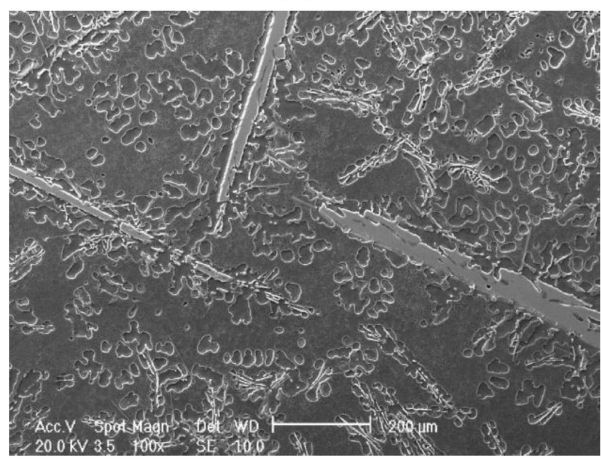

(b)

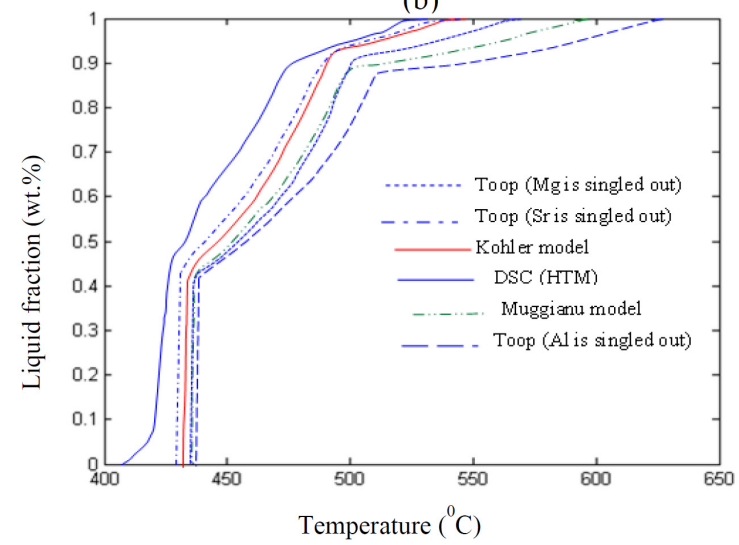

Figure 5. (a) SEM image and (b) Solidification curves of sample 3(6.88/27.67/65.45, Sr/Al/Mg, wt.\%). 


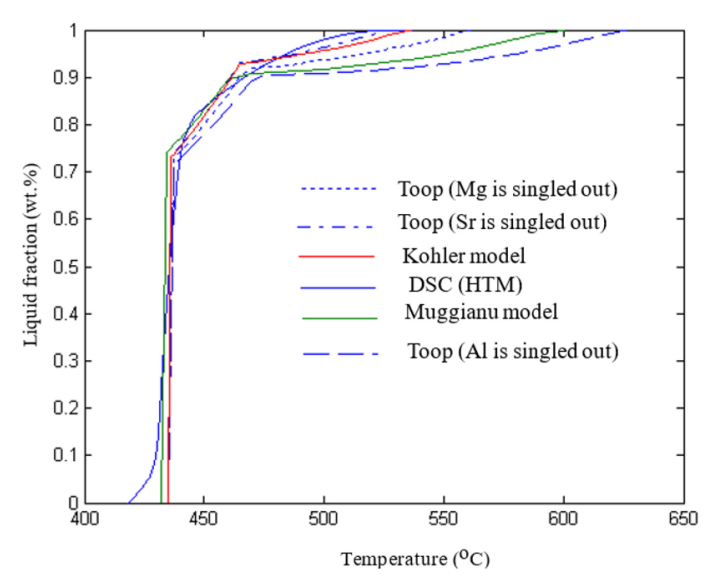

Figure 6. Solidification curves of sample 4 (4.56/31.63/63.81, Sr/ $\mathrm{Al} / \mathrm{Mg}$, wt.\%).

\section{Acknowledgment}

Authors would like to thank deanship of scientific research at the Hashemite university for their financial supports and prof. Mamoun Medraj form Concordia university his valuable comments.

\section{References}

1. Cahn RW. The coming of materials science. Oxônia, Reino Unido: Pergamon; 2003.

2. Kattner UR. Thermodynamic modeling of multicomponent phase diagram. JOM. 1997;49(12):14-9.

3. Haowei P, Zhiqiang H, Baicheng L. Study on dendritic growth in pressurized solidification of $\mathrm{Mg}-\mathrm{Al}$ Alloy using phase field simulation. J Mater Sci Technol. 2016;32(1):68-75.

4. Pelton AD. A general "geometric" thermodynamic model for multicomponent solutions. Calphad. 2001;25(2):319-28.

5. Kohler F. Estimation of the thermodynamic data for a ternary system from the corresponding binary systems. Monatsh Chem. 1960;91(4):738-40.

6. Muggianu YM, Gambino M, Bross JP. Enthalpies of formation of liquid alloys bismuth-gallium-tin at $723 \mathrm{~K}$ choice of an analytical representation of integral and partial excess functions of mixing. J Chim Phys. 1975;72(1):83-8.
7. Toop GW. Predicting ternary activities using binary data. Trans Metal Soc aime. 1965;233(5):850-5.

8. Hillert M. Empirical methods of predicting and representing thermodynamic properties of ternary solution phases. Calphad. 1980;4(1):1-12.

9. Ansara O. In thermochemistry in metallurgical chemistry. London: HMSO; 1972. p. 403-430.

10. Bernard AC, Kaufman L, Spender P. A comparison of calculated phase equilibria in selected ternary alloy systems using thermodynamic values derived from different models. Calphad. 1978;2(1):1-15.

11. Ansara O. Comparison of methods for thermodynamic calculation of phase diagram. Int. Met. Rev. 1979;24(1):20-53.

12. Lukas HL, Weiss J, Henig E.-Th. Strategies for the calculation of phase diagrams. Calphad. 1982;6(3):229-251.

13. Chartrand P, Pelton AD. On the choice of "geometric" thermodynamic models. J Phase Equilibria. 2000;21(2):141-7.

14. Qiao ZY, Xing X, Peng M. Thermodynamic criterion for judging the symmetry of ternary systems and criterion applications. $\mathrm{J}$ Phase Equilibria. 1996;17(6):502-7.

15. Mirkovic D, Grobner J, Fetzer RS, Kainer K. Solidification curves of AZ-magnesium alloys determined by DSC Experiments and Heat-transfer Model (DSC-HTM). In: Kainer KU. Magnesium: Proceedings of the 6th International Conference Magnesium Alloys and Their Applications. USA: Wiley Online Library; 2003. p. 842-847.

16. Hohne GW, Hemminger W, Flammershiem HJ. Differential scanning calorimetry: an introduction for practitioners. Heidelberg: Springer-Verlag Berlin; 1996.

17. Hemminger W, Cammenga H. Methoden der thermischen analyse. Heidelberg: Springer-Verlag Berli; 1989.

18. Chen SW, Huang CC. Solidification curves of Al-Cu, Al-Mg and Al-Cu- Mg alloys. Acta Mater. 1996;44:1955-65.

19. Aljarrah M, Medraj M. Thermodynamic assessment of the phase equilibria in the Al-Ca-Sr system using the modified quasichemical model. J Chem Thermodyn. 2008;40(4):724-34.

20. Aljarrah M, Medraj M. Thermodynamic modeling of the Mg$\mathrm{Ca}, \mathrm{Mg}-\mathrm{Sr}, \mathrm{Ca}-\mathrm{Sr}$ and $\mathrm{Mg}-\mathrm{Ca}-\mathrm{Sr}$ systems using the modified quasichemical model. Calphad. 2008;32(2):240-51.

21. Aljarrah M, Parvez MA, Essadiqi E, Li J, Medraj M. Microstructural characterization of Mg-Al-Sr alloys. Sci Technol Adv Mater. 2007;8(4):237-48.

22. Liu Z-T, Wang B-Y, Wang C, Zha M, Liu GJ, Yang Z-Z, et al. Microstructure and mechanical properties of Al-Mg-Si alloy fabricated by a short process based on sub-rapid solidification. J Mater Sci Technol. 2020;4(15):178-86. 